\title{
Reconnection and interchange instability in the near magnetotail
}

\author{
Joachim Birn ${ }^{1,2 *}$, Yi-Hsin Liu ${ }^{3}$, William Daughton ${ }^{4}$, Michael Hesse $^{5}$ and Karl Schindler ${ }^{6}$
}

\begin{abstract}
This paper provides insights into the possible coupling between reconnection and interchange/ballooning in the magnetotail related to substorms and flow bursts. The results presented are largely based on recent simulations of magnetotail dynamics, exploring onset and progression of reconnection. 2.5-dimensional particle-in-cell (PIC) simulations with different tail deformation demonstrate a clear boundary between stable and unstable cases depending on the amount of deformation, explored up to the real proton/electron mass ratio. The evolution prior to onset, as well as the evolution of stable cases, are governed by the conservation of integral flux tube entropy $S$ as imposed in ideal MHD, maintaining a monotonic increase with distance downtail. This suggests that ballooning instability in the tail should not be expected prior to the onset of tearing and reconnection. 3-D MHD simulations confirm this conclusion, showing no indication of ballooning prior to reconnection, if the initial state is ballooning stable. The simulation also shows that, after imposing resistivity necessary to initiate reconnection, the reconnection rate and energy release initially remain slow. However, when $S$ becomes reduced from plasmoid ejection and lobe reconnection, forming a negative slope in $S$ as a function of distance from Earth, the reconnection rate and energy release increase drastically. The latter condition has been shown to be necessary for ballooning/interchange instability, and the cross-tail structures that develop subsequently in the MHD simulation are consistent with such modes. The simulations support a concept in which tail activity is initiated by tearing instability but significantly enhanced by the interaction with ballooning/interchange enabled by plasmoid loss and lobe reconnection.
\end{abstract}

Keywords: Reconnection; Interchange; Magnetotail dynamics; Substorms

\section{Findings}

Particle-in-cell and MHD simulations of magnetotail dynamics demonstrate conservation of integral flux tube entropy during the simulated substorm growth phase. This indicates stability to ballooning prior to the onset of tearing and reconnection in the magnetotail. Plasmoid loss from reconnection, however, enables ballooning instability, which can enhance the reconnection rate and energy release and provide cross-tail structure.

\section{Introduction}

The mechanism(s) for the onset of magnetotail activity related to substorms or other dynamic events still represent an unsolved problem and a matter of controversy.

\footnotetext{
*Correspondence: jbirn@spacescience.org

1 Space Science Institute, Boulder, Colorado, USA

2 Guest Scientist, Los Alamos National Laboratory, Los Alamos, New Mexico, USA

Full list of author information is available at the end of the article
}

Crucial elements in this context are the tearing instability, presumably the initiator of magnetic reconnection and plasmoid formation (e.g., Schindler 1974), and ballooning/interchange instability (e.g., Liu et al. 2012, and references therein), which can provide cross-tail structure and wave modes that are possibly related to auroral onset arc features ("beading") observed prior to the expansion of the auroral oval (e.g., Keiling et al. 2013, and references therein).

The onset and growth of collisionless tearing instability depends strongly on the magnitude of the normal magnetic field $B_{z}$ (Brittnacher et al. 1995; Pellat et al. 1991; Schindler 2007) and on the current sheet thickness (e.g., Daughton 1999). Through force balance along the tail axis, a reduction of the normal magnetic field $B_{z}$ is closely coupled to an increase of the current density (Hesse and Birn 2000). Therefore, the onset of tearing requires sufficient current sheet thinning, or the formation of a thin embedded current sheet.

\section{Springer}


The onset of tearing and reconnection, however, may be preceded by the onset of another instability, such as the ballooning instability (e.g., Liu et al. 2012, and refences therein), which operates even under ideal MHD constraints. As shown by Schindler and Birn (2004), ballooning/interchange instability is closely related to the properties of a flux tube entropy function (Birn et al. 2009; Wolf et al. 2009)

$$
S=\int p^{1 / \gamma} d s / B
$$

where the integral is taken along closed field lines. Simply stated, a tail configuration is stable to ideal MHD ballooning modes when $S$ increases monotonically downtail. This is typical of the quiet magnetotail. Integral flux tube entropy conservation also plays a crucial role in the evolution toward thin current sheet formation and eventual loss of equilibrium (Birn et al. 2009). In this paper, we review and summarize recent findings on the onset of tearing and the role of ballooning/interchange modes, based on 2.5-dimensional particle-in-cell (PIC) simulations (Liu et al. 2014) and 3-dimensional MHD simulations (Birn et al. 2011).

\section{General procedure}

Both PIC and MHD simulations are based on a similar concept. The simulations start from an equilibrium tail configuration that is stable to ballooning and tearing. This configuration is gradually deformed by adding magnetic flux to the tail, which causes current sheet thinning and eases the conditions for the onset of tearing. In contrast to some other driving approaches, the amount of added flux is kept finite, such that the driving subsides and the tail may also settle into a new equilibrium if the deformation is insufficient to initiate instability. For larger deformations, the PIC simulations naturally lead to the onset of tearing instability, whereas the onset of ballooning is inhibited by the 2-D constraint. However, it is possible to test the stability criterion, namely the variation of the entropy function $S$.

In contrast, the 3-D MHD simulations permit investigating the occurrence and effects of ballooning, particularly when plasmoid loss and lobe reconnection change the entropy function and stability. However, they need imposing a dissipation mechanism (here, finite resistivity) to initiate reconnection. In the next section, we summarize major findings of the PIC simulations, while the following section is devoted to MHD results. For more details of the codes, initial, and boundary conditions, we refer to Liu et al. (2014) and Birn et al. (2011).

\section{PIC simulations of reconnection onset}

The PIC simulations start from a 2-D initial state (Hesse and Schindler 2001; Liu et al. 2014) (Fig. 1a), which is gradually deformed by the addition of magnetic flux to the lobes through the high-latitude boundaries. The parameter $a$ measures the amount of this added flux or of the deformation of the field, which decreases tailward from the near-Earth boundary. Several ion/electron mass ratios were used, up to the real proton/electron mass ratio $m_{i} / m_{e}=1836$. Depending on the magnitude of $a$ and the mass ratio, the configuration either remained stable, although compressed, or became unstable to tearing and reconnection.

Figure 1, modified after Figure two of Liu et al. (2014), illustrates the evolution of stable and unstable runs. Panel (a) shows the initial state, which is the same for all runs. The corresponding monotonic entropy function $S(x)$ is shown in panel (b), demonstrating the initial stability to ballooning. The following two panels show the current density and magnetic field structure for two runs with mass ratios $m_{i} / m_{e}=400$, (c) for $a=3.33$ after relaxation into a new equilibrium, and (d) for $a=5$ after the onset of reconnection. The third row panels (e, f) show the characteristic current sheet half-thickness $L_{z}$ and the bottom panels $\left(\mathrm{g}, \mathrm{h}\right.$ ) the $B_{z}$ values as function of time, evaluated at the location where $B_{z}$ reaches a local minimum or the current density maximizes along the $x$ axis, at $x \sim-13,(\mathrm{e}, \mathrm{g})$ for $a=2.5$ and (f, h) for $a=5$.

The evolution of the characteristic parameters, as well as the final configuration of the stable runs (left), appears independent of the mass ratio. The onset of tearing and reconnection occurs in the PIC simulation when $B_{z}$ and the current sheet thickness are sufficiently reduced to demagnetize electrons. The clearest indication of instability onset is shown by the parameter $B_{z}$ (panel h). The evolution prior to onset is basically independent of the mass ratio. However, the curves of $B_{z}$ for $m_{i} / m_{e}=25-$ 400 show a clear transition from this early, approximately linear decrease, to a much more rapid decrease, while the curve for $m_{i} / m_{e}=1836$ indicates stability for this deformation parameter. Liu et al. (2014) chose the last point before the deviation from the higher mass ratio curves (which show onset at a later time or, for $m_{i} / m_{e}=1836$ and $a=5$, not at all) as the onset point, indicated by small circles.

Based on this identification, onset parameters were determined, shown in Fig. 2 as function of the mass ratio for different deformation amplitudes $a$ : (a) the current sheet half-thickness $L_{z}$ at the location of the minimum of $B_{z}$, (b) the normal magnetic field $b=B_{z \min } / B_{0}$. Vertical bars correspond to unstable cases with onset times and locations identified as described above. Crosses represent stable cases. Due to the driving, these cases also lead to increases in current density and decreases in current sheet thickness and $B_{z}$ in the near tail region. In these cases the parameters were evaluated at late times, when the configuration approached a new equilibrium, as shown in 


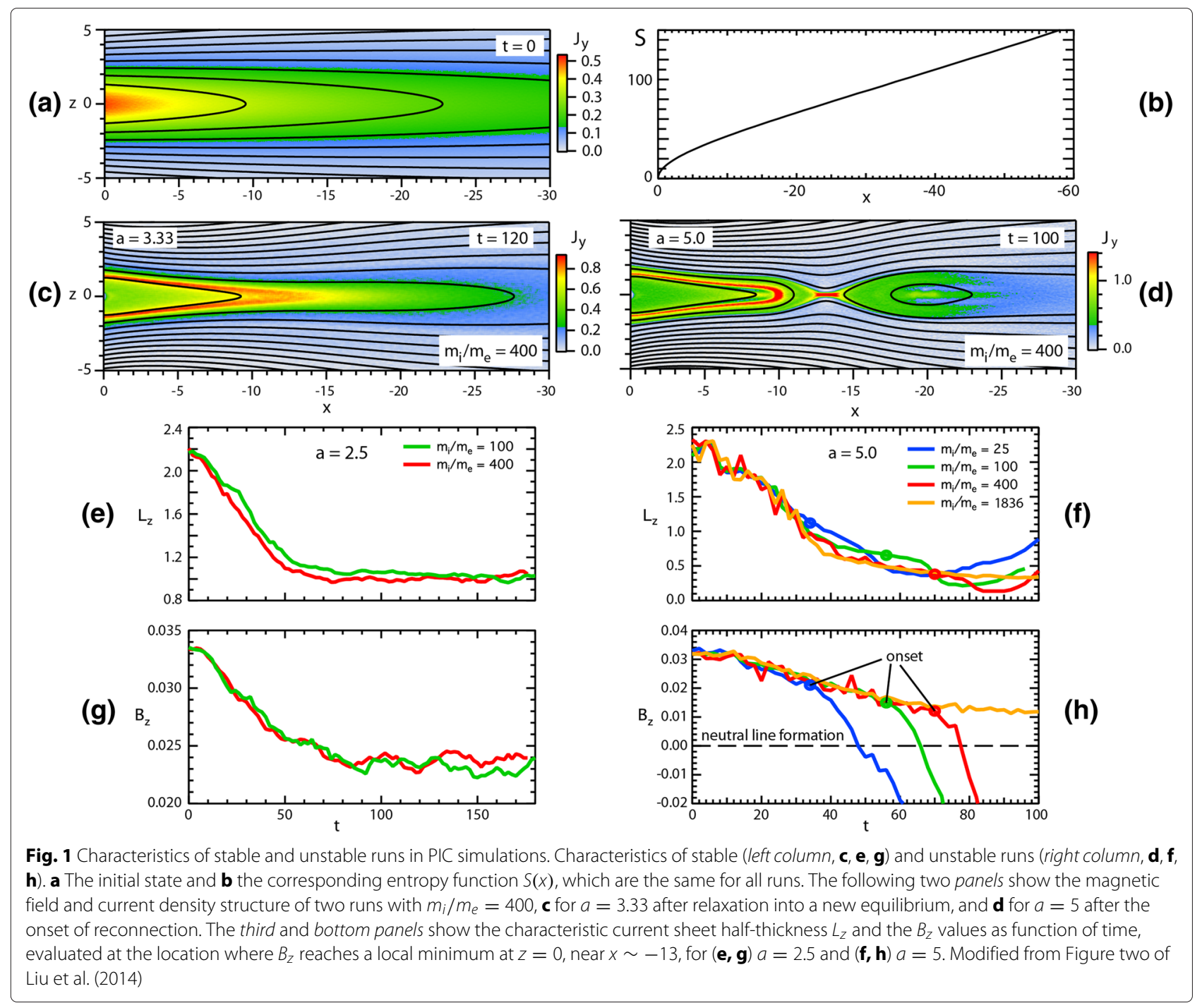

Fig. 1c, choosing the location of the local maximum of $\delta B_{z}=\left|B_{z}-B_{z}(t=0)\right|$ along the $x$ axis. Figure 2 indicates a well-defined stability transition, which shows a power law dependence on the mass ratio, consistent with electron tearing (Hesse and Schindler 2001). We note that, for larger deformation with $a=7.5$, the real mass ratio case also becomes unstable, and that the stability transition parameters $L_{z}$ and $b$ can reasonably well be inferred by the power law extrapolation (dashed lines) from the lower mass ratio cases. This is important because in many cases (particularly in 3-D) PIC simulations at $m_{i} / m_{e}=1836$ are still either unfeasible or very expensive.

Evaluating the onset problem and the evolution of reconnection at different mass ratios $m_{i} / m_{e}$ also provides important insights into the physical mechanisms of onset and fast reconnection, as well as of the validity and usefulness of simpler approaches, such as MHD. The fact that the onset parameters depend on the mass ratio (dashed lines in Fig. 2) supports identification of the electron tearing instability as the onset mode (rather than the ion tearing mode, which should not depend on the electron mass) (Liu et al. 2014). In contrast, the evolution prior to the onset, the final states of stable cases, as well as the fast reconnection after $x$-line formation are largely independent of $m_{i} / m_{e}$. These are important clues for the validity and applicability of an MHD approach prior to onset and to identify the final state (Zaharia and Birn 2007), and for the dominance of ion dynamics during the phase of fast reconnection (Birn and et al. 2001).

To investigate the potential instability to ballooning modes, Birn and Hesse (2014) and Liu et al. (2014) evaluated the properties of the entropy function (1), which for 2-D equilibria with $\mathbf{B}=\nabla A \times \hat{\mathbf{y}}$ should be a function of the flux variable $A$, constant along field lines. The result is shown in Fig. 3 (bottom), together with characteristic states (top), for one stable (left) and two unstable cases 


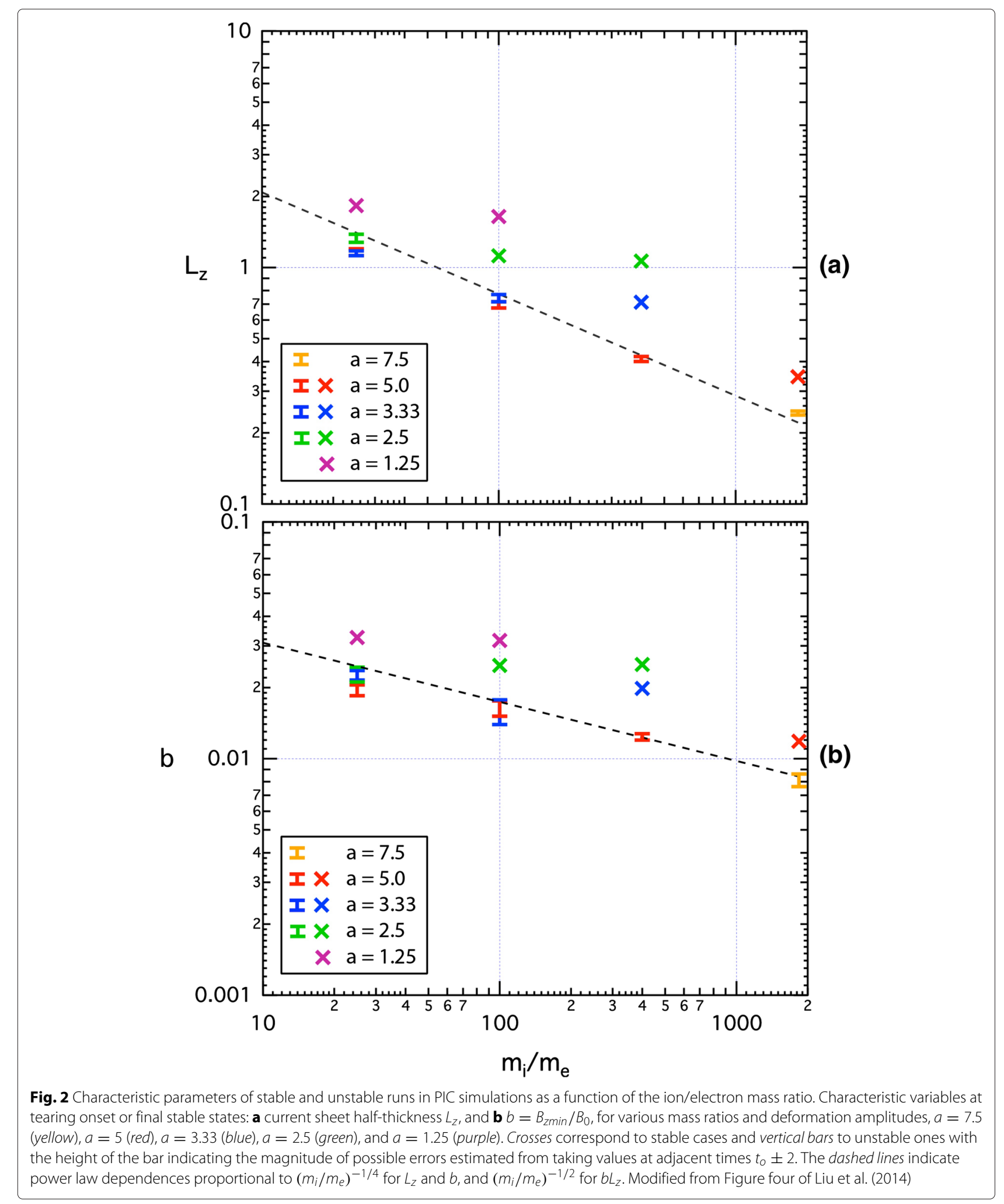

(center and right). To make a connection to the MHD case, the pressure was defined by the trace of the full tensor, including both ions and electrons; however, similar curves were also found for the individual pressures. Labels $a-d$ indicate significant points: point $a$ marks the first reconnected field line, point $b$ the X-point, point $c$ the farthest 


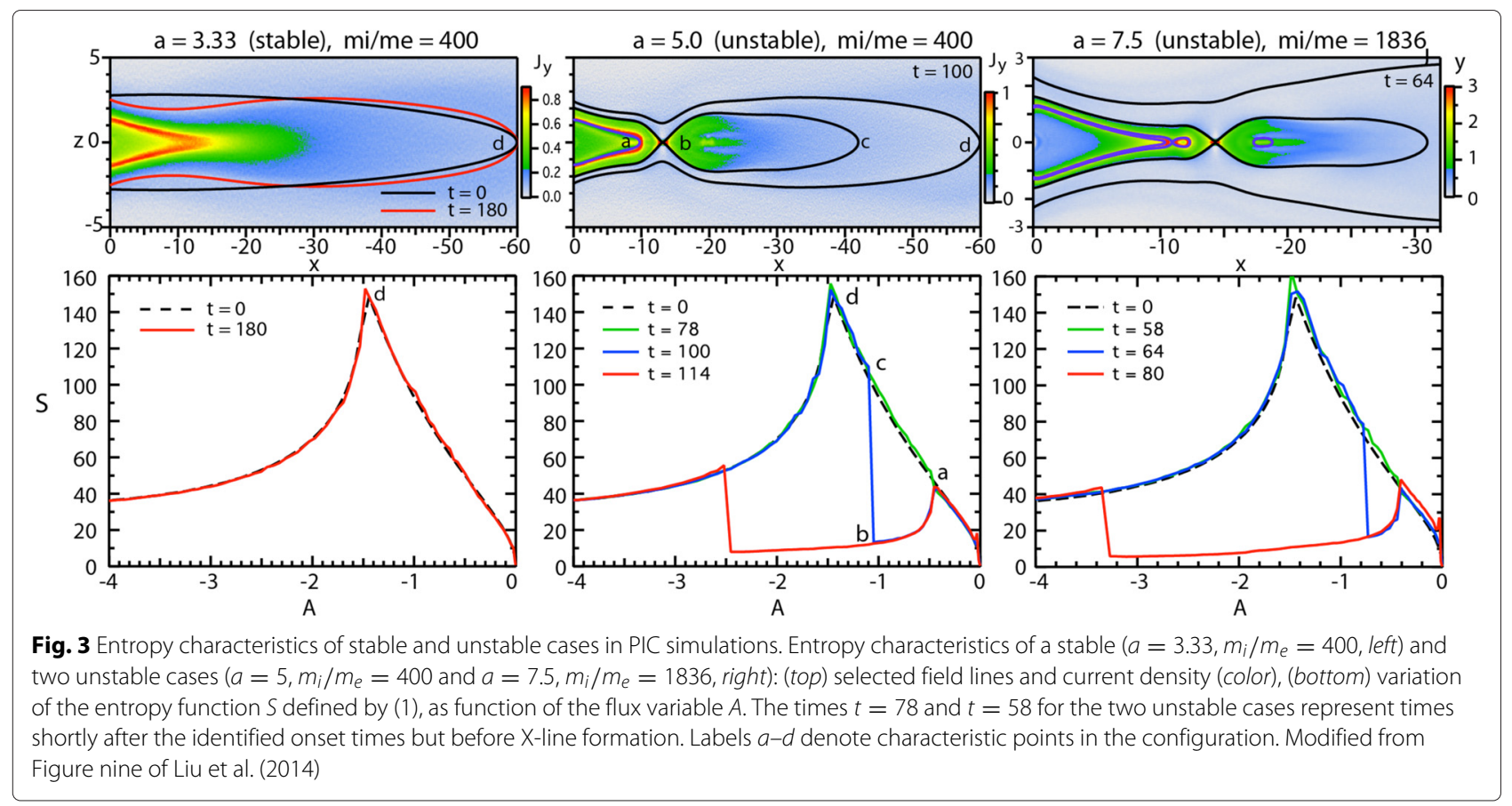

point of the separatrix connected to the X-point, while point $d$ indicates the last closed field line. The integral (1) was taken from one foot point at the left boundary to the other for closed field lines. For open field lines with $A<A_{d} \approx-1.5$, irrelevant for the stability concept, the integral was taken from the left to the right boundary, adding top and bottom parts for continuity.

The intensified current sheet in the stable case (top left in Fig. 3) represents an equilibrium structure, whereas the intensified current layers earthward of the reconnection site (top center and right in Fig. 3) in the unstable cases represent dipolarization fronts (Nakamura and et al. 2002), sharp increases in $B_{z}$ associated with fast earthward flows, documented also in earlier PIC simulations (e.g., Sitnov et al., 2009, Birn and Hesse, 2014). The field structure of the $m_{i} / m_{e}=1836$ case (top right in Fig. 3) shows a small island earthward of the main X-line, which exists for a brief period $\left(\sim 2-3 \Omega_{c i}^{-1}\right)$ after neutral line formation. Such island structures earthward of the main reconnection site were first postulated by Schindler (1974) and later found in many observations embedded in earthward flows (e.g., Slavin et al. 2003; Walsh et al. 2007).

Figure 3 demonstrates, for $m_{i} / m_{e}=400$ and $m_{i} / m_{e}=$ 1836 , that the entropy function is well conserved for stable cases, as well as for unstable ones prior to the onset of reconnection, despite significant pressure changes. The conservation of the entropy function $S(A)$ prior to onset, and particularly the conservation of monotonicity for closed field lines with $A>A_{d}$, indicate that the tail configuration remains stable to ballooning (Schindler and Birn 2004) prior to the onset of tearing. The entropy function $S(A)$ is also conserved for reconnected flux tubes after reconnection. However, the shortening of flux tubes by plasmoid formation makes the entropy function non-monotonic and thus potentially unstable to ballooning. This will be addressed further in the following section, on the basis of 3-D MHD simulations.

\section{MHD results}

The MHD simulation (Birn et al. 2011) discussed here was based on a 3-D initial tail equilibrium that included a transition toward a dipole field. It spans a region $0 \geq$ $x \geq-60$, with one spatial unit corresponding to about $1.5 R_{E}$ and inner boundary at $x_{G S M} \approx-7.5 R_{E}$. The configuration also included a net cross-tail magnetic field $B_{y}$ of $\sim 1 \%$ of the lobe field strength, breaking exact mirror symmetry but having no significant effect on the evolution. Similar to the 2-D PIC simulations, the onset and dynamic phase was preceded by a driven phase $\left(t<t_{0}\right.$, with $t_{0}=61$ in the chosen dimensionless units of $\sim 10 \mathrm{~s}$.), during which a finite amount of magnetic flux was added at the high-latitude boundaries, causing compression and the formation of a thin current sheet, embedded in the wider plasma sheet. In contrast to the PIC simulations, the ideal MHD approach does not include a natural transition to unstable tearing modes. Therefore, a finite, spatially localized, resistivity was imposed after $t=t_{0}$; without resistivity, the configuration remained stable. The resistivity peaked at $x=-8$, close to the location of maximum current intensification, decaying with distance $s$ proportional to $1 / \cosh ^{2} s$ on scales of 4,8 , and 2 in $x, y$ and $z$, 
respectively. Reconnection did not start until $t \approx 90$, but it remained weak until $t \approx 120$.

Figure 4 illustrates the subsequent evolution, showing the cross-tail electric field $E_{y}$ (color) in the $y=0$ plane, together with magnetic flux contours. (The contours are not exactly field lines because of the abovementioned small cross-tail $B_{y}$ component.) The electric field increases drastically after $t \approx 120$, and even more in the earthward collapsing, dipolarizing field.

Figure 5 further documents this rapid increase through the temporal change of the reconnected flux, integrated over the area in the $x, y$ plane where $B_{z}$ has turned negative, due to reconnection. The decrease of the rate of reconnected flux after $t=121$ in Fig. 5 coincides with the stopping of the associated flow burst near the inner boundary and a tailward bounce, which is found in observations as well (Ohtani et al. 2004; Panov and et al. 2010).

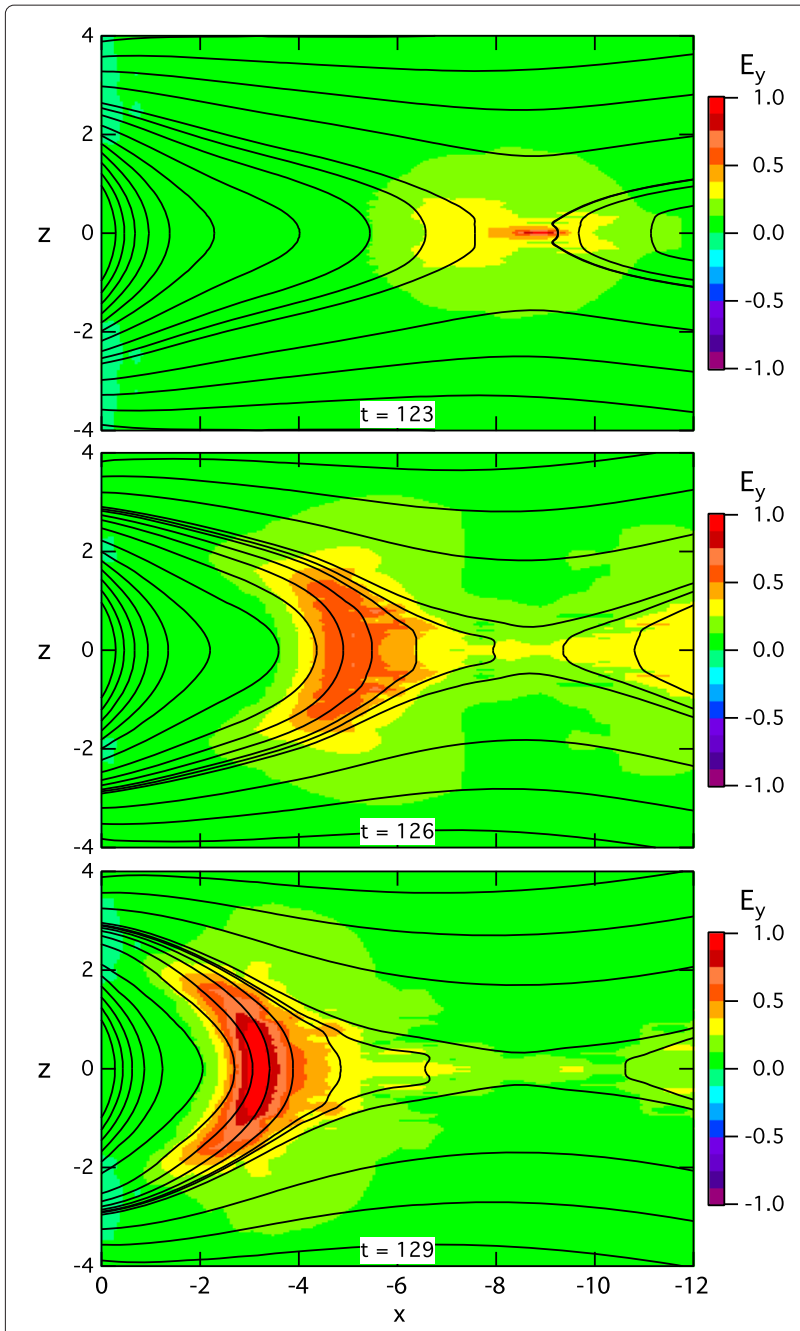

Fig. 4 Cross-tail electric field in the MHD simulation. Cross-tail electric field $E_{y}$ (color) in the $x, z$ plane, obtained from a 3-D MHD simulation of tail reconnection and dipolarization (Birn et al. 2011)

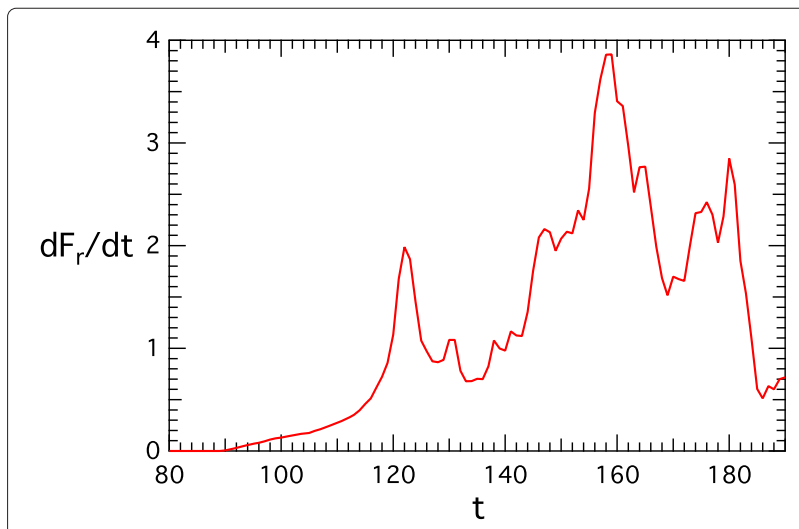

Fig. 5 Evolution of reconnected flux in the MHD simulation. Temporal change of reconnected flux obtained from the 3-D MHD simulation (Birn et al. 2011)

The subsequent increase is related to additional reconnection intensifications and flow bursts after $t \approx 140$, that arise off center at $y \neq 0$, and decay also when the flows are stopped nearer to Earth. These bursts are possibly related to the growth of ballooning, introducing structure in $y$ and feedback on a wavelength comparable to the vortex structure on the outside of the first flow burst.

The cross-tail structure with different flow bursts is demonstrated by Fig. 6, which shows the entropy integral $S$ (color), defined by (1), for field lines crossing the $z=0$ plane at $t=155$ and $t=185$, together with velocity vectors and $B_{z}$ contours (solid black lines). Figure 6 clearly shows the association of the earthward flow bursts with low-entropy flux tubes (blue), originally postulated by Pontius and Wolf (1990), which accumulate near the dipolar region on the left.

The close relation between the onset of faster reconnection, flow bursts, and the reduction of the entropy function is further illustrated by Fig. 7, which shows the entropy integral $S$ as function of the crossing point through the $x$ axis, for different times. (Note that the numerical values of the dimensionless quantity $S$ cannot be compared directly with the PIC values in Fig. 3, because of different normalization and differences in the underlying tail equilibrium, such as $3-\mathrm{D}$ versus $2-\mathrm{D}$ and higher background pressure in the PIC simulation.) The driven evolution prior to $t=61$, not shown here, changes the function $S(x)$ slightly, due to motions along the $x$ axis associated with the thin current sheet formation and reduction in $B_{z}$. This, however, does not change the field line entropy and the monotonicity of $S(x)$; the entropy of the last closed field line, for instance, remains conserved to better than $0.1 \%$. It is obvious that the slope remains positive until about $t=110$ but changes to negative at $t=120$ (and afterwards). This further supports the view that the system has become unstable to ballooning/interchange. 


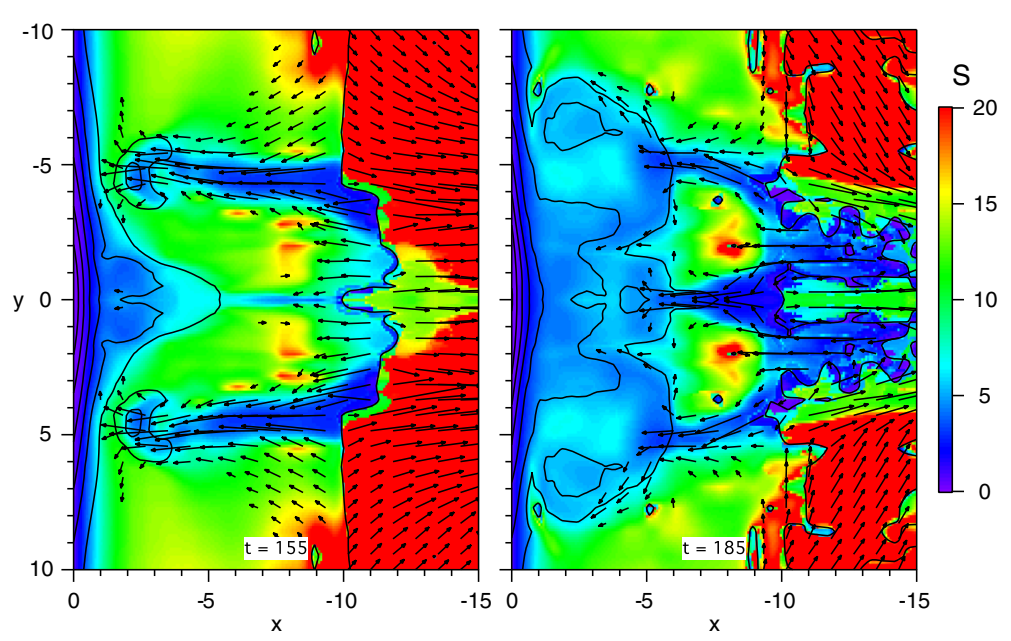

Fig. 6 Entropy function $S$ in the $z=0$ plane in the MHD simulation. Entropy quantity (1) in the equatorial plane at two different times; modified from Figure eight of Birn et al. (2011). Arrows show velocity vectors with $v>100 \mathrm{~km} / \mathrm{s}$, and solid black lines represent $B_{z}=$ const. contours; the one farthest to the right is the $B_{z}=0$ contour

Fast flows, however, are produced also in 2-D reconnection without involvement of interchange instability. To further test the effects of the 3-D structure, and to demonstrate that more flux is transferred and more energization occurs when additional ballooning/interchange instability occurs, we have taken the fields in the $y=0$ plane from the 3-D simulation as the starting configuration for a 2-D MHD simulation, imposing the same resistivity. Figure 8 shows a comparison of characteristic quantities between the 2-D and 3-D results, (a) the reconnected flux, and (b) the kinetic energy. The 3-D results were evaluated in a 2-D fashion at the $z=0$ plane or, as shown here, averaged over a vicinity of this plane. (The differences were minor.) The early evolution up to $t \approx 120$ is very similar, due to the fact that the 3-D results show little variation with $y$. After

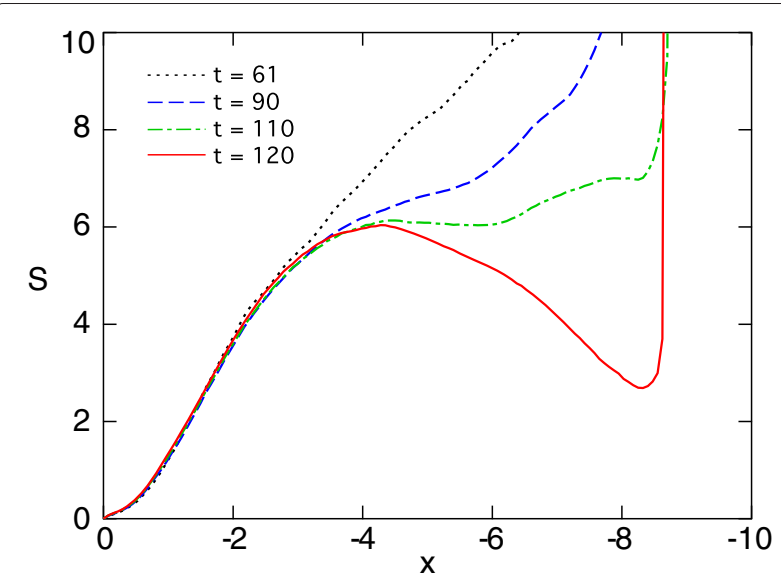

Fig. 7 Entropy variation in the MHD simulation. Variation of the entropy integral $S(x)$ along the $x$ axis at different times, obtained from the 3-D MHD simulation; modified from Figure nine of Birn et al. (2011) $t=120$, the reconnected flux and the total kinetic energy rise much more rapidly in the 3-D case and reach larger total values. It is interesting to note that the reconnected flux in the 2-D and 3-D case at first settle at a similar level but then the 3-D case takes off again. This has to do with

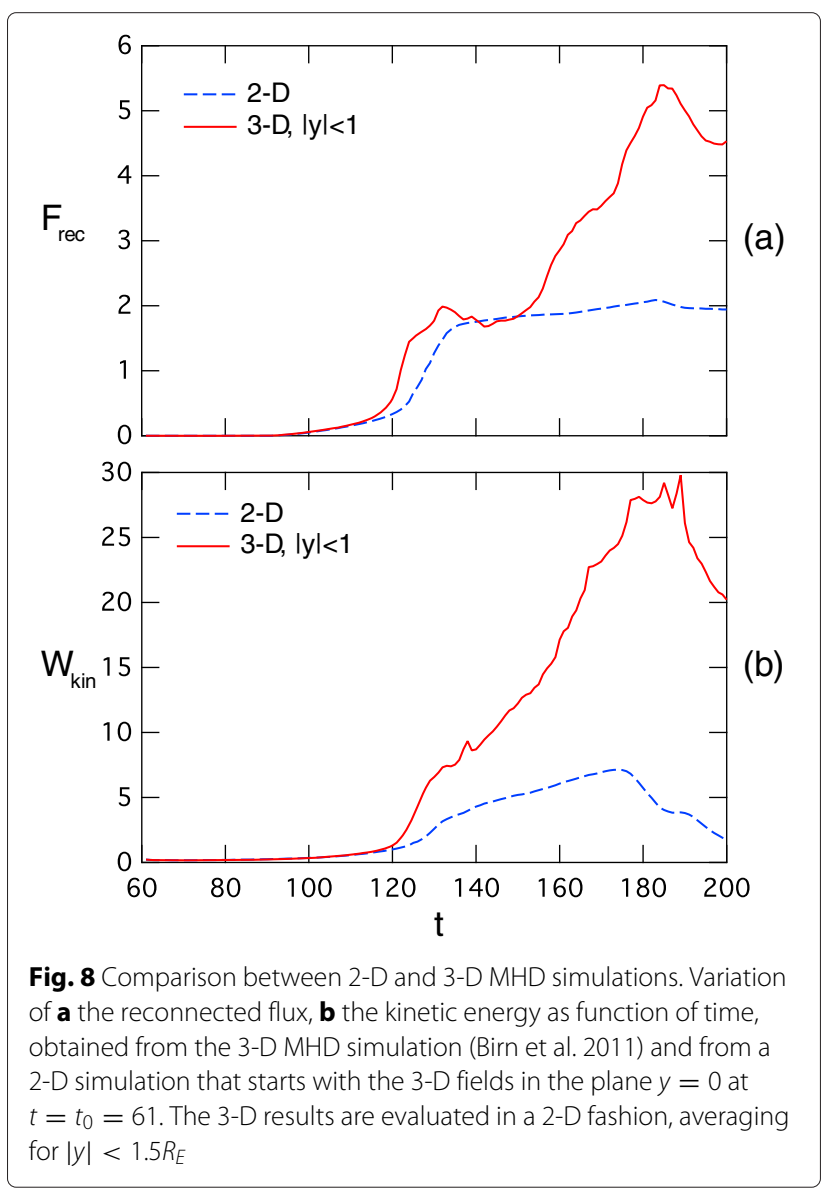


secondary reconnection near $z=0$, visible in Fig. 6, which does not occur in the 2-D case.

\section{Summary and conclusions}

We have presented recent results on the stability and possible interaction of the major modes discussed in the context of substorms and other tail activity: tearing and ballooning/interchange. Two-dimensional particle-in-cell simulations of tearing and reconnection onset under conditions of slow tail deformation indicate a sharp boundary between stable and unstable cases, dependent on the deformation amplitude and ion/electron mass ratio (Liu et al. 2014). The evolution prior to onset was found independent of the mass ratio, governed by integral entropy conservation as imposed in MHD, consistent with earlier PIC simulation results obtained for $m_{i} / m_{e}=100$ (Birn and Hesse 2014). As a consequence, the entropy variation along the tail remains monotonically increasing, despite the fact that the equatorial $B_{z}$ develops a local minimum, associated with a tailward gradient. Since the entropy variation governs ballooning/interchange stability in 2-D configurations (Schindler and Birn 2004), this result suggests that ballooning instability in the tail should not be expected from slow adiabatic evolution prior to the onset of tearing and reconnection, although it cannot be excluded that ballooning might arise in the inner more dipole-like region under suitable conditions.

3-D MHD simulations (Birn et al. 2011) confirm this conclusion, showing no indication of ballooning prior to reconnection, if the initial state is ballooning stable. The simulation also shows that, after imposing resistivity necessary to initiate reconnection, the reconnection rate and energy release initially remain slow, but increase drastically when $S$ becomes reduced from plasmoid ejection and lobe reconnection and develops a negative slope as function of distance. The latter condition has been shown to be necessary for ballooning/interchange instability, and the cross-tail structures that develop subsequently in the MHD simulation are consistent with such modes. The simulations support a concept in which tail activity is initiated by tearing instability but significantly enhanced by the interaction with ballooning/interchange enabled by plasmoid loss and lobe reconnection.

\section{Competing interests}

The authors declare that they have no competing interests.

\section{Authors' contributions}

JB carried out the MHD simulations and drafted the manuscript. YHL, WD, and $\mathrm{MH}$ carried out the PIC simulations discussed, and KS was responsible for the stability analysis. All authors participated in the interpretation of the results, read, and approved the manuscript.

\section{Acknowledgements}

Part of this work was performed at Los Alamos under the auspices of the US Department of Energy, supported by the NSF's GEM and by NASA's MMS/SMART Theory and Modeling, SR\&T and Heliophysics Theory Programs.
The simulations were performed using resources from the Los Alamos Institutional Computing Program, with further resources from the Blue Waters sustained-petascale computing project, which is supported by the NSF (OCI 07-25070) and the state of Illinois.

\section{Author details}

${ }^{1}$ Space Science Institute, Boulder, Colorado, USA. ${ }^{2}$ Guest Scientist, Los Alamos National Laboratory, Los Alamos, New Mexico, USA. ${ }^{3}$ NASA/Goddard Space Flight Center, Greenbelt, Maryland, USA. ${ }^{4}$ Los Alamos National Laboratory, Los Alamos, New Mexico, USA. ${ }^{5}$ NASA/Goddard Space Flight Center, Greenbelt, Maryland, USA. ${ }^{6}$ Ruhr-University, Bochum, Germany.

Received: 1 May 2015 Accepted: 25 June 2015

Published: 16 July 2015

\section{References}

Birn J, et al. (2001) Geospace Environment Modeling (GEM) magnetic reconnection challenge. J Geophys Res 106:3715

Birn J, Hesse M, Schindler K, Zaharia S (2009) Role of entropy in magnetotail dynamics. J Geophys Res 114:A00D03. doi:10.1029/2008JA014,015

Birn J, Nakamura R, Panov E, Hesse M (2011) Bursty bulk flows and dipolarization in MHD simulations of magnetotail reconnection. J Geophys Res 116:A01210. doi:10.1029/2010JA016083

Birn J, Hesse M (2014) Forced reconnection in the near magnetotail: onset and energy conversion in PIC and MHD simulations. J Geophys Res:119. doi:10.1002/2013JA019354

Brittnacher M, Quest KB, Karimabadi H (1995) A new approach to the linear theory of single-species tearing in two-dimensional quasi-neutral sheets. J Geophys Res 100:3551

Daughton W (1999) The unstable eigenmodes of a neutral sheet. Phys. Plasmas 6:1329

Hesse M, Birn J (2000) Near- and mid-tail current flow during substorms: Small-scale and large-scale aspects of current disruption. In: Ohtani S, Fujii R, Hesse M, Lysak RL (eds). Magnetospheric Current Systems, Geophys. Monogr. American Geophysical Union, Washington, DC Vol. 118. p 295

Hesse M, Schindler K (2001) The onset of magnetic reconnection in the magnetotail. Earth Planets Space 53:645

Keiling A, Shiokawa K, Uritsky V, Sergeev V, Zesta E, Kepko L, Østgaard N (2013) Auroral signatures of the dynamic plasma sheet. In: Keiling A, Donovan E, Bagenal F, Karlsson T (eds). Auroral Phenomenology and Magnetospheric Processes: Earth And Other Planets, Geophys. Monogr. American Geophysical Union, Washington, DC Vol. 197. pp 317-336. doi:10.1029/2012GM001231

Liu WW, Liang J, Donovan EF, Spanswick E (2012) If substorm onset triggers tail reconnection, what triggers substorm onset?. J Geophys Res 117(A11). doi:10.1029/2012JA018161

Liu Y-H, Birn J, Daughton W, Hesse M, Schindler K (2014) Onset of reconnection in the near magnetotail: PIC simulations. J Geophys Res 119:1-17. doi:10.1002/2014JA020492

Nakamura R, et al. (2002) Motion of the dipolarization front during a flow burst event observed by Cluster. Geophys Res Lett 29(4):1942

Ohtani SI, Shay MA, Mukai T (2004) Temporal structure of the fast convective flow in the plasma sheet: comparison between observations and two-fluid simulations. J Geophys Res 109:A03210. doi:10.1029/2003JA010002

Panov EV, et al. (2010) Multiple overshoot and rebound of a bursty bulk flow. Geophys Res Lett 37:L8103. doi:10.1029/2009GL041971

Pellat R, Coroniti FV, Pritchett PL (1991) Does ion tearing exist? Geophys Res Lett 18:143

Pontius DH Jr., Wolf RA (1990) Transient flux tubes in the terrestrial magnetosphere. Geophys Res Lett 17:49

Schindler K (1974) A theory of the substorm mechanism. J Geophys Res 79:2803

Schindler K, Birn J (2004) MHD stability of magnetotail equilibria including a background pressure. J Geophys Res 109:A10208. doi:10.1029/2004JA010537

Schindler K (2007) Space Plasma Activity. Cambridge, England

Sitnov MI, Swisdak M, Divin AV (2009) Dipolarization fronts as a signature of transient reconnection in the magnetotail. J Geophys Res 114:A04202. doi:10.1029/2008JA013980

Slavin JA, et al. (2003) Geotail observations of magnetic flux ropes in the plasma sheet. J Geophys Res 108:1015. doi:10.1029/2002JA009557 
Walsh AP, Fazakerley AN, Wilson RJ, Alexeev IV, Henderson PD, Owen CJ, Lucek E, Carr C, Dandouras I (2007) Near-simultaneous magnetotail flux rope observations with Cluster and Double Star. Ann Geophys 25(8):1887-1897 Wolf RA, Wan Y, Xing X, Zhang J-C, Sazykin S (2009) Entropy and plasma sheet transport. J Geophys Res 114:A00D05. doi:10.1029/2009JA014044

Zaharia S, Birn J (2007) Adiabatic plasma equilibria and application to a reconnection problem. Phys Plasmas 14:07:210

\section{Submit your manuscript to a SpringerOpen ${ }^{\circ}$ journal and benefit from:}

- Convenient online submission

Rigorous peer review

- Immediate publication on acceptance

- Open access: articles freely available online

- High visibility within the field

- Retaining the copyright to your article

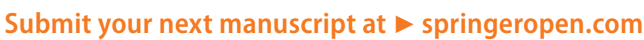

